\begin{tabular}{|c|l|}
\hline Title & Discharge phenomena in a cavitation bubble induced by liquid-phase laser ablation \\
\hline Author(s) & Sasaki, Koichi; Takahashi, Y uta \\
\hline Citation & $\begin{array}{l}\text { Journal of Physics D : A pplied Physics, 50(32), 325202 } \\
\text { https://doi.org/10.1088/1361-6463/aa754a }\end{array}$ \\
\hline Issue Date & 2017-08-16 \\
\hline Doc URL & http://hdl.handle.net/2115/71311 \\
\hline Type & article(author version) \\
\hline File Information & Cavitation_Discharge3.pdf \\
\hline
\end{tabular}

Instructions for use 


\title{
Discharge phenomena in a cavitation bubble induced by liquid-phase laser ablation
}

\author{
Koichi Sasaki and Yuta Takahashi \\ Division of Quantum Science and Engineering, Hokkaido University, Kita 13, Nishi 8, \\ Kita-ku, Sapporo 060-8628, Japan \\ E-mail: sasaki@qe.eng.hokudai.ac.jp
}

\begin{abstract}
We observed the discharge phenomena in a cavitation bubble induced by liquid-phase laser ablation. A remarkable result was the deformation of the cavitation bubble. The formation of a swelling was observed from the cavitation bubble when the gas-liquid boundary reached a close distance from a needle electrode. The growth direction of the swelling was perpendicular to the gas-liquid boundary. The discharge toward the center of the target occurred when the swelling connected the electrode and the cavitation bubble. These experimental results suggest the perpendicular electric field to the gas-liquid boundary of the cavitation bubble. In other words, the cavitation bubble induced by liquid-phase laser ablation is conductive and it has free charges.
\end{abstract}

\section{Introduction}

This work has two backgrounds. One is the growing interest in electrical discharges involving liquid phases [1-3]. Electrical discharges with liquids are categorized into two types. The first type is gas-phase discharges in contact with liquids. This is realized by employing a plasma jet [4-8] which is placed above the liquid surface or by using the liquid surface as an electrode of the discharge [9-16]. The second type is electrical discharges in bubbles in liquids. A widely used method is just depositing an electrical power into a liquid [17-23]. The power deposition into the liquid produces bubbles via the Joule heating, and plasmas are produced in the bubbles by electrical discharges. The second type can enhance the contacting surface between the plasma and the liquid, resulting in the efficient transport of radicals produced in the plasma to the liquid. However, just depositing the power into the liquid has difficulty in fundamental investigations on discharge mechanisms in bubbles, since the thermal production of bubbles occurs randomly. A bubble produced at a fixed position and a fixed timing is useful to carry out the efficient investigation on the discharge phenomena in a bubble [24-27].

Another background is an open question about the colloidal solution synthesized

by liquid-phase laser ablation. Recently, liquid-phase laser ablation is utilized for 
synthesizing various nanoparticles without using chemicals [28,29]. In this technique, a solid-state target installed in a liquid is irradiated by intense laser pulses [30]. Atomic and molecular species are ejected from the target by the laser irradiation, resulting in the formation of nanoparticles that are stored in the liquid as the form of a colloidal solution $[31,32]$. The open question is related to the fact that the colloidal solution thus synthesized has a high zeta potential. This means nanoparticles have a large amount of electrical charges, but the origin of the charges has not been clarified yet.

In this work, we observed electrical discharges in a cavitation bubble induced by liquid-phase laser ablation. The cavitation bubble is a remarkable phenomenon of liquid-phase laser ablation, and is induced as a result of the abrupt formation of a laser-produced plasma in the liquid [33-35]. The cavitation bubble has the dynamics of expansion, shrinkage, and collapse. Since the position and the timing of the bubble formation are determined strictly by the irradiation of the laser pulse, it is useful for the fundamental investigation on discharge phenomena in bubbles. The present experimental results depict the discharge processes between an electrode and the ablation target via the cavitation bubble. In addition, the experimental results indicate that the electric field is perpendicular to the gas-liquid boundary of the cavitation bubble. In other words, the cavitation bubble induced by liquid-phase laser ablation stores a large amount of free charges. The experimental results are consistent with a model calculation which assumes free charges on the gas-liquid boundary. Since it has been shown that the growth field of nanoparticles in liquid-phase laser ablation is the inside of the cavitation bubble [36-40], it is suggested that the origin of the high zeta potential of the colloidal solution synthesized by liquid-phase laser ablation is the electrical charges stored in the cavitation bubble.

\section{Experimental method}

Figure 1(a) shows the experimental apparatus. In this apparatus, a Nd:YAG laser was used for generating a cavitation bubble in water, while an electrical discharge was formed in the cavitation bubble. We prepared a rectangular vessel which was filled with distilled water at room temperature. The water had neutral $\mathrm{pH}$, and its electrical conductivity was estimated to be $1.6 \times 10^{-3} \mathrm{~S} / \mathrm{m}$. A titanium or alumina $\left(\mathrm{Al}_{2} \mathrm{O}_{3}\right)$ target was attached on a target holder which was installed in water. The target holder was connected to a motor and was rotated to disperse the ablation point. The target holder was electrically grounded. The target was irradiated by focused Nd:YAG laser pulses at a wavelength of $1.06 \mu \mathrm{m}$ from the normal direction. The duration of the Nd:YAG laser pulse was roughly $10 \mathrm{~ns}$. The region in front of the ablation point was illuminated by a metal halide lamp, and the pattern of the transmitted lamp light was captured using a highspeed camera. This shadowgraph imaging gave us the location of the cavitation bubble and the temporal variation of the bubble size after the irradiation of the Nd:YAG laser pulse.

We installed a needle electrode at a distance from the ablation point as shown in 
Fig. 1(b). The tip of the electrode was separated from the gas-liquid boundary of the cavitation bubble, even when the bubble size became maximum. The electrode was a molybdenum rod with a diameter of $0.3 \mathrm{~mm}$. The molybdenum rod was covered with an alumina tube, and the length of the molybdenum rod exposed to water was $2 \mathrm{~mm}$. The needle electrode was connected to a dc or pulsed power supply via a resistance of $50 \mathrm{k} \Omega$. The maximum current of the dc power supply was $100 \mathrm{~mA}$, and the maximum power of the pulsed power supply was $300 \mathrm{~W}$. The discharge occurred between the needle electrode and the target via the cavitation bubble. The optical emission from the discharge channel was captured using the same high-speed camera as that used in the shadowgraph imaging. The electrical potentials at the both sides of the resistance were measured using high-voltage probes, and the current passing through the electrode was obtained from the difference in the two potentials and the magnitude of the resistance.

\section{Results}

Figure 2 shows the temporal variation of the radius of the cavitation bubble. A titanium target was used in this experiment, and the energy of the laser pulse was $230 \mathrm{~mJ}$. The radius shown in Fig. 2 is defined by the distance between the target surface and the leading edge of the cavitation bubble. The horizontal axis in Fig. 2 is the delay time $t_{\mathrm{D}}$ after the irradiation of the Nd:YAG laser pulse. As shown in Fig. 2, the bubble radius expanded rapidly after the irradiation of the $\mathrm{Nd}$ : $\mathrm{YAG}$ laser pulse. The expansion speed was decelerated with $t_{\mathrm{D}}$. The bubble began to shrink after the maximum size at a delay time of $t_{\mathrm{D}} \simeq 280 \mu \mathrm{s}$, and the bubble size became minimum at $t_{\mathrm{D}} \simeq 580 \mu \mathrm{s}$. This phenomenon is called the collapse of the cavitation bubble. It is noted here that the size of the collapsed cavitation bubble is not identified in this experiment because of the insufficient temporal resolution $(25 \mu \mathrm{s})$ of the high-speed movie captured at a flame rate of $40 \mathrm{kHz}$. The second cavitation bubble with a smaller radius was formed after the collapse. In addition, the third cavitation bubble was formed after the collapse of the second cavitation bubble.

The discharge between the needle electrode and the titanium target is depicted in Fig. 3(a). The pictures show the shadows of the cavitation bubble and the needle electrode, in addition to the discharge channel which is seen as the bright spot in the pictures. The time below each picture shows the delay time $t_{\mathrm{D}}$ after the irradiation of the Nd:YAG laser pulse. A dc power supply at a voltage of $5 \mathrm{kV}$ was used in this experiment. The discharge gap is represented by a switch in the equivalent circuit shown in Fig. 3(b). The resistance through the plasma is represented by $R_{\mathrm{p}}$, while $R_{\mathrm{ET}}$ shows the resistance between the electrode and the target through water and the cavitation bubble. Figure 3(c) shows the temporal variation of the current which was obtained from the difference between the potentials $V_{\mathrm{A}}$ and $V_{\mathrm{B}}$. The roman numbers illustrated in Fig. 3(c) correspond to the delay times of the pictures shown in Fig. 3(a). The electrode voltage $\left(V_{\mathrm{B}}\right)$ was decreased to $0.6 \mathrm{kV}$ during the discharge, and the electrical energy consumed by $R_{\mathrm{p}}$ was estimated to be $\sim 10 \mathrm{~mJ}$. 
As shown in Fig. 3(a), the distance between the electrode and the gas-liquid boundary of the cavitation bubble became shorter with the delay time because of the expansion of the cavitation bubble. The distance between the tip of the electrode and the nearest gas-liquid boundary was approximately $1.5 \mathrm{~mm}$ at $t_{\mathrm{D}}=50 \mu \mathrm{s}$ as shown in picture (i) of Fig. 3(a). It is noted here that the current shown in Fig. 3(c) increased slightly in the period of $50 \leq t_{\mathrm{D}} \leq 165 \mu \mathrm{s}$. At a delay time of $t_{\mathrm{D}}=165 \mu \mathrm{s}$, when the distance between the tip of the electrode and the nearest gas-liquid boundary was approximately $0.4 \mathrm{~mm}$, as shown in picture (ii) of Fig. 3(a), we observed the formation of a swelling from the cavitation bubble. The swelling was lengthened toward the electrode. We observed the discharge when the electrode and the cavitation bubble was connected by the lengthened swelling, as show in picture (iii) of Fig. 3(a). As shown in Fig. 3(c), the discharge stopped at $t_{\mathrm{D}}=510 \mu \mathrm{s}$, when the cavitation bubble and the electrode was separated by water as shown in picture (v) of Fig. 3(a).

Figure 4 shows the discharge channels when the tip of the electrode was positioned at three different points. The target was a titanium plate. It is clearly shown in Fig. 4 that the lengthening direction of the swelling was always perpendicular to the gas-liquid boundary of the cavitation bubble. In addition, the discharge channel was always perpendicular to the gas-liquid boundary, and the discharge channel connected the electrode and the center of the cavitation bubble, even though a shorter discharge length was possible if it was not directed to the center of the cavitation bubble.

Figure 5 shows a similar experimental result to that shown in Fig. 3, but in this case we employed an alumina target and a pulsed power supply. The pulsed high voltage was applied at $t_{\mathrm{D}}=107 \mu \mathrm{s}$, and we observed a spiky displacement current at this timing. The current before the discharge was negligible as shown in Fig. 5(b) since the electrode and the grounded target holder was almost insulated by the alumina target. As shown in Fig. 5(a), we observed similar experimental results to those shown in Fig. 3, namely, we observed the formation and the perpendicular lengthening of the swelling from the cavitation bubble. The discharge channel was directed to the center of the cavitation bubble. The pulsed power supply was not essential to obtain this experimental result. Similar results were obtained when we used the dc power supply and the alumina target.

\section{Discussion}

The experimental results clearly show the deformation of the cavitation bubble at a close distance $(<1 \mathrm{~mm})$ between the tip of the electrode and the gas-liquid boundary. This is completely different from the experimental results reported by Foster and Gucker [26,27]. They observed the discharge in a cavitation bubble produced by applying an ultrasonic power to water. In their results, the cavitation bubble kept the spherical shape even when a needle electrode is located at a close distance from the gas-liquid boundary. It is speculated that the electric field in the experiment of Foster and Gucker was higher than our experiment, since they observed the discharge in the bubble even though the electrode was separated from the bubble by water. We believe that the most important 
difference of our experimental condition from the work by Foster and Gucker is the method for producing the cavitation bubble.

The present experimental results suggest that the cavitation bubble produced by liquid-phase laser ablation is conductive. An experimental result which suggests the conductive cavitation bubble is shown in Fig. 3(c). We observed slight increase in the current passing through $R_{\mathrm{RT}}$ in the period of $50 \leq t_{\mathrm{D}} \leq 165 \mu \mathrm{s}$. In this period, the cavitation bubble expanded as shown in Fig. 3(a). If we assume that the cavitation bubble is not conductive, the electrical resistance $R_{\mathrm{ET}}$ between the electrode and the target should become larger because of the increase in the path length through water. However, experimental result shows the decrease in $R_{\mathrm{ET}}$ with $t_{\mathrm{D}}$, which reveals that a part of the current passed through the cavitation bubble.

A more remarkable experimental result which suggests conductive cavitation bubble is that both the lengthening of the swelling and the discharge channel were always perpendicular to the gas-liquid boundary, as shown in Fig. 4. This result strongly suggests that the gas-liquid boundary of the cavitation bubble works as a conductive surface, and the electric field is perpendicular to the gas-liquid boundary. Another possibility is the influence of the crater at the center of the cavitation bubble. The crater is formed by the erosion of the target by laser ablation, and there is a possibility that the crater enhances the electric field in the vicinity of it. To check the influence of the ablation crater, we carried out the experiment shown in Fig. 6. In this experiment, we formed a deep ablation crater by irradiating laser pulses before the discharge experiment. The deep crater, which is shown by the red spot in Fig. 6, is located at a distance of 1.5 $\mathrm{mm}$ from the center of the cavitation bubble, and the distance between the electrode and the deep crater is closer than that between the electrode and the center of the cavitation bubble. As shown in Fig. 6, we observed the discharge channel toward the center of the cavitation bubble even in this experimental condition. This means that the discharge channel is not influenced by the crater. Therefore, the experimental result shown in Fig. 4 suggests that the electric field is perpendicular to the gas-liquid boundary of the cavitation bubble, and the gas-liquid boundary works as a conductive surface.

We have examined the possibility of the formation of the swelling from the cavitation bubble by evaluating the two forces on the gas-liquid boundary. One is the electrostatic pressure which is calculated as a function of the distance between the gas-liquid boundary and the tip of the electrode, under the assumption that the gasliquid boundary of the cavitation bubble is conductive. Figure 7 shows the simplified model which we used in the theoretical estimation of the electrostatic pressure. The gas-liquid boundary is spherical in the reality, but we assume a flat boundary for the sake of simplicity. This is a reasonable approximation when the bubble radius is much longer than the distance between the electrode and the gas-liquid boundary. Negative charges are induced on the gas-liquid boundary in Fig 7 if it is conductive, resulting in the attractive force between the electrode and the gas-liquid boundary. The electrostatic capacitance of the system shown in Fig. 7 is calculated by basic electrostatics, and is 
given by

$$
C=\frac{2 \pi \varepsilon l}{\ln \left(\frac{l}{a} \sqrt{\frac{4 d+l}{4 d+3 l}}\right)},
$$

where $\varepsilon$ is the permittivity of water, $d$ is the distance between the tip of the electrode and the gas-liquid boundary, and $l$ and $a$ are the length and the radius of the electrode, respectively $(l=2 \mathrm{~mm}$ and $a=0.15 \mathrm{~mm}$ ). This approximated expression is valid when $a \ll d, l$. If we assume that the charges are concentrated at the tip of the electrode, the electric field at the position $\mathrm{O}$ in Fig. 7 is approximated by

$$
E=\frac{C V}{2 \pi \varepsilon d^{2}}
$$

where $V$ is the applied voltage to the electrode. The electrostatic pressure is obtained by

$$
P_{\mathrm{E}}=\frac{1}{2} \varepsilon E^{2}
$$

This pressure attracts the gas-liquid boundary toward the electrode.

The other force on the gas-liquid boundary is due to the difference between the water pressure and the pressure inside the cavitation bubble. It is well known that the pressure inside the expanded cavitation bubble is lower than the pressure in the surrounding water [41], and the gas-liquid boundary is attracted toward the center of the cavitation bubble. The pressure difference between the bubble and the water is given by Rayleigh-Plesset equation [41], and is

$$
P_{\mathrm{B}}-P_{0}=\rho R(t) \frac{\mathrm{d}^{2} R(t)}{\mathrm{d} t^{2}}+\frac{3}{2}\left(\frac{\mathrm{d} R(t)}{\mathrm{d} t}\right)^{2}+\frac{4 \nu}{R(t)} \frac{\mathrm{d} R(t)}{\mathrm{d} t}+\frac{2 S}{R(t)},
$$

where $P_{\mathrm{B}}$ and $P_{0}$ are the bubble and water pressures, respectively, $R(t)$ denotes the temporal variation of the bubble radius, and $\rho, \nu$, and $S$ are the weight density, the kinematic viscosity coefficient, and the surface tension of water, respectively. The second and third terms in the right hand side of Eq. (4) are negligible when the bubble size is almost maximum because of the slow expansion speed $(\mathrm{d} R(t) / \mathrm{d} t \simeq 0)$. In addition, the first term dominates the forth one in the expanded bubble. Therefore, we can estimate $P_{\mathrm{B}}-P_{0}$, when the bubble size is almost maximum, by substituting the temporal variation of $R(t)$ shown in Fig. 2 into Eq. (4).

Figure 8 shows the relationship between $P_{\mathrm{E}}$ and $d$. Here we assumed $V=5 \mathrm{kV}$. The horizontal dotted line shows the value of $P_{0}-P_{\mathrm{B}}$. Although this is a rough estimation on the basis of the aforementioned assumptions, Fig. 8 reveals the possibility of bubble deformation when $d \simeq 0.5 \mathrm{~mm}$ since the electrostatic pressure $P_{\mathrm{E}}$ exceeds $P_{\mathrm{B}}-P_{0}$. This result roughly agrees with our experimental observation on the formation of the swelling from the cavitation bubble. In other words, the assumption of the conductive cavitation bubble explains the experimental observation. The amount of electrical charges estimated by this calculation is $10^{-8} \mathrm{C}$, and the corresponding number of charges is on the order of $10^{10}$. On the other hand, if the liquid-phase laser-ablation plasma has an electron density of $10^{19} \mathrm{~cm}^{-3}$ [42], it has a total charges of $10^{15}$ if we assume 
a volume of $0.1 \mathrm{~mm}^{3}$. Therefore, $10^{10}$ charges are a realistic value as the residue from the laser ablation plasma. It is noted finally that the charges are not provided from the electrical ground, since we observed the similar experimental results when we employed an alumina target, as shown in Fig. 5. The charges are considered to be originated from the laser ablation plasma.

\section{Conclusions}

In this work, we observed the discharge phenomena in a cavitation bubble induced by liquid-phase laser ablation. We observed the formation of a swelling from the cavitation bubble at a close distance between the electrode and the gas-liquid boundary. The discharge occurred when the swelling connected the electrode and the cavitation bubble. The experimental results suggest that the cavitation bubble is conductive and it has free charges. This may be a unique feature of the cavitation bubble induced by liquid-phase laser ablation, since the deformation of the cavitation bubble is not observed when it is produced by an ultrasonic power [26,27]. Since it has been found that the growth field of nanoparticles in liquid-phase laser ablation is the inside of the cavitation bubble [36-40], the present experimental results may explain the origin of the high zeta potential of the colloidal solution synthesized by liquid-phase laser ablation. 
[1] Bruggeman P J, Kushner M J, Locke B R, Gardeniers J G E, Graham W G, Graves D B, HofmanCaris R C H M, Maric D, Reid J P, Ceriani E, Rivas D F, Foster J E, Garrick S C, Gorbanev Y, Hamaguchi S, Iza F, Jablonowski H, Klimova E, Kolb J, Krcma F, Lukes P, Machala Z, Marinov I, Mariotti D, Thagard S M, Minakata D, Neyts E C, Pawlat J, Petrovic Z L, Pflieger R, Reuter S, Schram D C, Schröter S, Shiraiwa M, Tarabová B, Tsai P A, Verlet J R, von Woedtke T, Wilson K R, Yasui K and Zvereva G 2016 Plasma Sources Sci. Technol. 25053002

[2] Locke B R, Sato M, Sunka P, Hofman M R and Chang J S 2006 Ind. Eng. Chem. Res. 45882

[3] Bruggeman P J and Leys C 2009 J. Phys. D: Appl. Phys. 42053001

[4] Teschke M, Kedzierski J, Finatu-Dinu E G, Korzec D and Engemann J 2005 IEEE Trans. Plasma Sci. 33310

[5] Schulz-von der Gathen V, Schaper L, Knake N, Reuter S, Niemi K, Gans T and Winter J 2008 J. Phys. D: Appl. Phys. 41194004

[6] Kolb J F, Mohamed A A H, Price R O, Swanson R J, Bowman A, Chiavarini R L, Stacey M and Schoenbach K H 2008 Appl. Phys. Lett. 92241501

[7] Urabe K, Morita T, Tachibana K, and Ganguly B N 2010 J. Phys. D: Appl. Phys. 43095201

[8] Algwari Q T and O'Connell D 2011 Appl. Phys. Lett. 99121501

[9] Hayashi D, Hoeben W F L M, Dooms G, van Veldhuizen E M, Rutgers W R and Kroesen G M W 2000 J. Phys. D: Appl. Phys. 332769

[10] Mezei P and Cserfalvi T 2007 Appl. Spectrosc. Rev. 42573

[11] Bruggeman P J, Ribezl E, Maslani A, Degroote J, Malesevic A, Rego R, Vierendeels J and Leys C 2008 Plasma Source Sci. Technol. 17025012

[12] Bruggeman P J, Liu J J, Degroote J, Kong M G, Vierendeels J and Leys C 2008 J. Phys. D: Appl. Phys. 41215201

[13] Shirai N, Nakazawa M, Ibuka S and Ishii S 2008 IEEE Trans. Plasma Sci. 361160

[14] Shirai N, Nakazawa M, Ibuka S and Ishii S 2009 Jpn. J. Appl. Phys. 48036002

[15] Shirai N, Ichinose K, Uchida S, and Tochikubo F 2011 Plasma Sources Sci. Technol. 20034013

[16] Rumbach P, Bartels D M, Sankaran R M and Go D B 2015 Nature Comm. 67248

[17] Stalder K R, Woloszko J, Brown I G and Smith C D 2001 Appl. Phys. Lett. 794503

[18] Sato K and Yasuoka K 2008 IEEE Trans. Plasma Sci. 361144

[19] Bruggeman P, Degroote J, Vierendeels J and Leys C 2008 Plasma Sources Sci. Technol. 17025008

[20] Ishijima T, Hotta H and Sugai H, Appl. Phys. Lett. 91121501

[21] Ishijima T, Sugiura H, Saito R, Toyoda H and Sugai H 2010 Plasma Sources Sci. Technol. 19 015010

[22] Hussein R O, Nie X, Northwood D O, Yerokhin A and Matthews A 2010 J. Phys. D: Appl. Phys. 43105203

[23] Shirafuji T and Himeno Y 2013 Jpn. J. Appl. Phys. 52 11NE03

[24] Tachibana K, Takekata Y, Mizumoto Y, Motomura H and Jinno M 2011 Plasma Sources Sci. Technol. 20034005

[25] Tian W, Tachibana K and Kushner M J 2014 J. Phys. D: Appl. Phys. 47055202

[26] Gucker S N, Sommerer T J and Foster J E 2014 IEEE Trans. Plasma Sci. 422636

[27] Foster J E, Sommers B, and Gucker S 2015 Jpn. J. Appl. Phys. 54 01AF05

[28] Barcikowski S and Compagnini G 2013 Phys. Chem. Chem. Phys. 153022

[29] Barcikowski S, Devesa F and Moldenhauer K 2009 J. Nanopart. Res. 111883

[30] Sasaki K and Takada N 2010 Pure Appl. Chem. 821317

[31] Mafune F, Kohno J, Takeda Y, Kondow T, and Sawabe H 2000 J. Phys. Chem. B 1049111

[32] Mafune F, Kohno J, Takeda Y, Kondow T, and Sawabe H 2011 J. Phys. Chem. B 1055114

[33] Tsuji T, Okazaki Y, Tsuboi Y and Tsuji M 2007 Jpn. J. Appl. Phys. 461533

[34] Tsuji T, Thang D-H, Okazaki Y, Nakanishi M, Tsuboi Y and Tsuji M 2008 Appl. Surf. Sci. 254 5224

[35] Sasaki K, Nakano T, Soliman W, and Takada N 2009 Appl. Phys. Express 2046501

[36] Soliman W, Takada N, and Sasaki K 2010 Appl. Phys. Express 3035201 
[37] Ibrahimkutty S, Wagener P, Menzel A, Plech A and Barcikowski S 2012 Appl. Phys. Lett. 101 103014

[38] Wagener P, Ibrahimkutty S, Menzel A, Plech A and Barcikowski S 2013 Phys. Chem. Chem. Phys. 153068

[39] Ibrahimkutty S, Wagener P, Rolo T S, Karpov D, Menzel A, Baumbach T, Barcikowski S and Plech A 2015 Sci. Rep. 516313

[40] Takeuchi M and Sasaki K 2016 Appl. Phys. A 122312

[41] Brennen C 1995 Cavitation and Bubble Dynamics (Oxford University Press, Oxford)

[42] Amans D, Chenus A-C, Ledoux G, Dujardin C, Reynaud C, Sublemontier O, Masenelli-Varlot K, and Guillois O 2009 Diamond Relat. Mater. 18177 


\section{Figure captions}

Fig.1 Experimental setup. (a) shows the whole of the apparatus and (b) illustrates the geometry of the electrode, the target, and the cavitation bubble.

Fig.2 Temporal evolution of the radius of the cavitation bubble. The laser energy was $230 \mathrm{~mJ}$.

Fig.3 (a) shows the electrode, the cavitation bubble, and the discharge channel observed at five delay times after laser ablation. The simplified equivalent circuit is shown in (b), and (c) is the temporal variation of the current which was obtained from $V_{\mathrm{A}}-V_{\mathrm{B}}$ in (b). The roman numbers illustrated in (c) correspond to those in (a).

Fig.4 Discharge channels observed at three different positions of the electrode.

Fig.5 (a) Discharge channel and (b) voltage and current waveforms when an alumina target was employed.

Fig.6 Discharge channel when an ablation crater was located at the red spot in the figure.

Fig.7 Simplified discharge geometry which was assumed in the theoretical calculation of the electrostatic pressure.

Fig.8 Electrostatic pressure as a function of the distance between the tip of electrode and the gas-liquid boundary of the cavitation bubble. The horizontal dotted line shows the pressure difference between the cavitation bubble and water. 


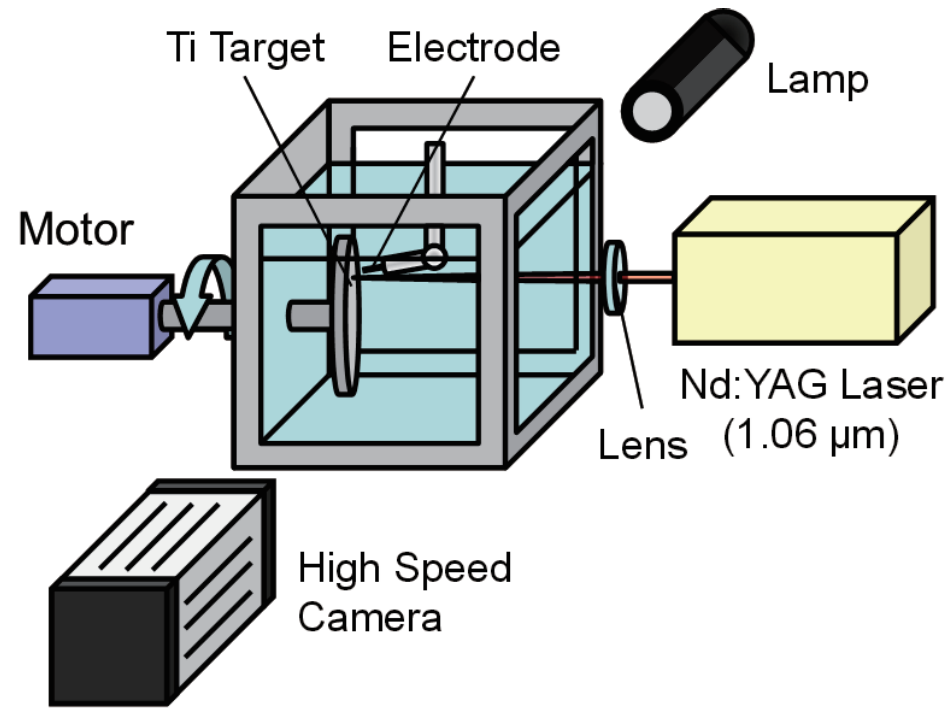

(a)

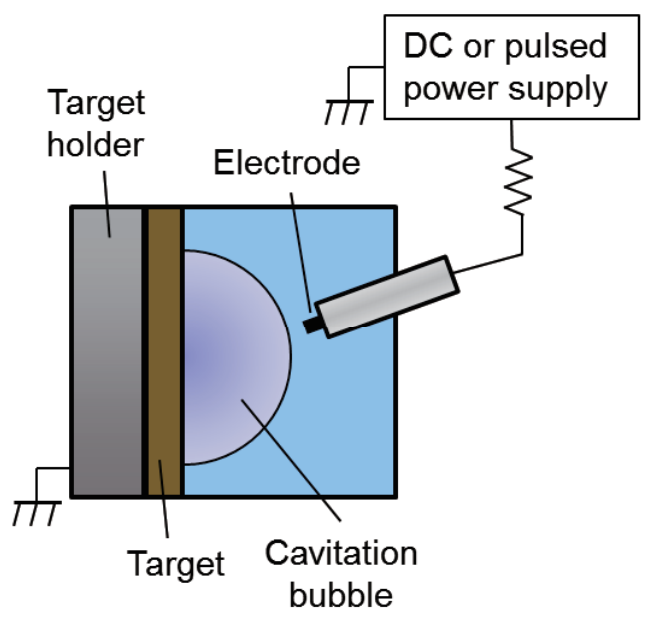

(b)

Figure 1. Experimental setup. (a) shows the whole of the apparatus and (b) illustrates the geometry of the electrode, the target, and the cavitation bubble. 


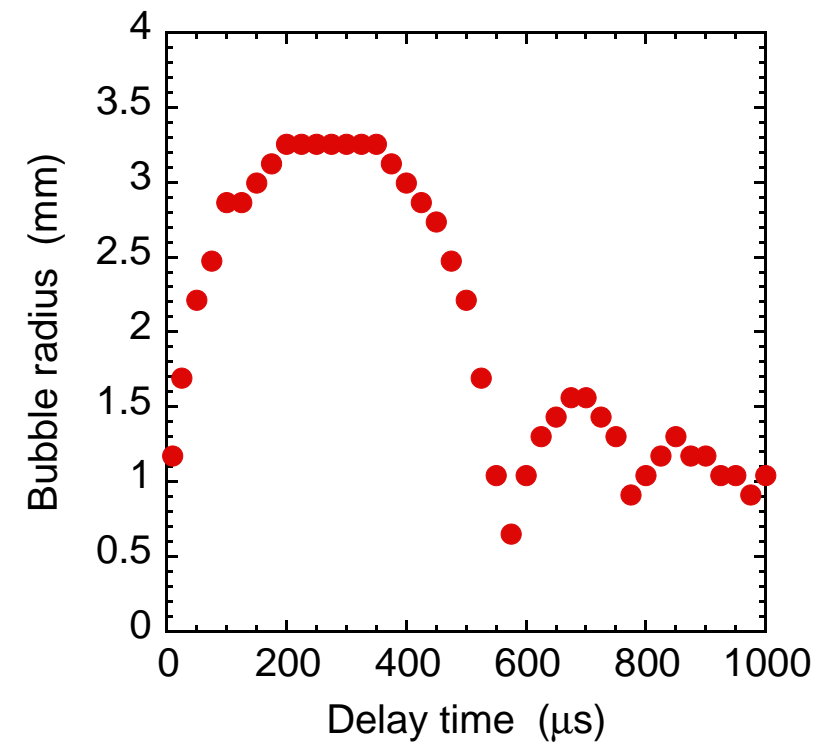

Figure 2. Temporal evolution of the radius of the cavitation bubble. The laser energy was $230 \mathrm{~mJ}$. 


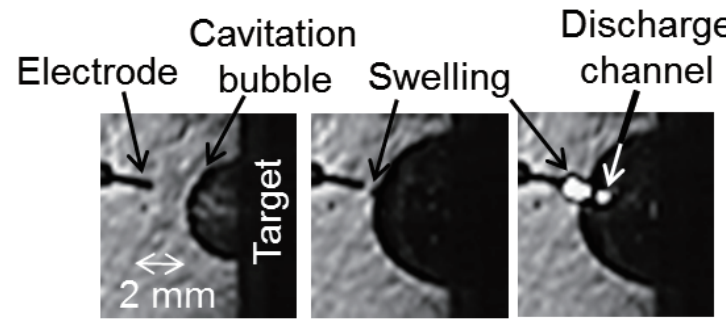
(i) $50 \mu \mathrm{s}$
(ii) $165 \mu \mathrm{s}$
(iii) $170 \mu \mathrm{s}$
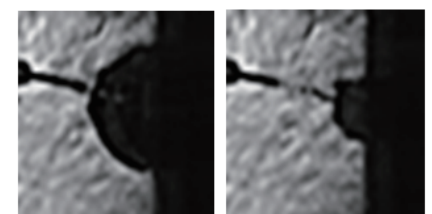

(iv) $430 \mu \mathrm{s} \quad$ (v) $510 \mu \mathrm{s}$

(a)

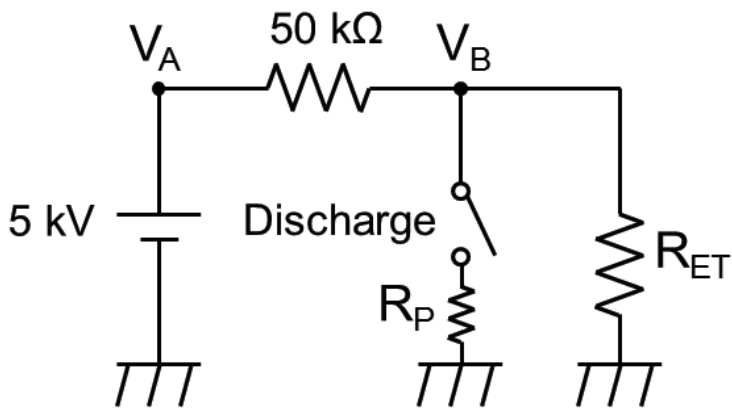

(b)

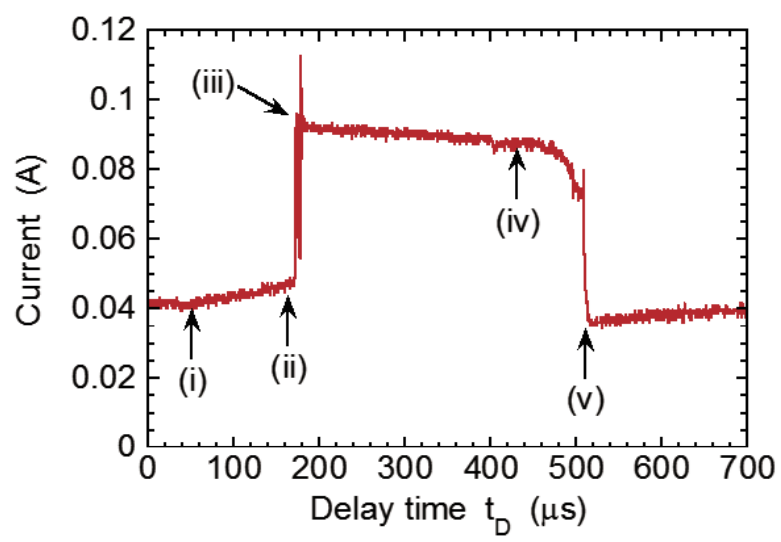

(c)

Figure 3. (a) shows the electrode, the cavitation bubble, and the discharge channel observed at five delay times after laser ablation. The simplified equivalent circuit is shown in (b), and (c) is the temporal variation of the current which was obtained from $V_{\mathrm{A}}-V_{\mathrm{B}}$ in (b). The roman numbers illustrated in (c) correspond to those in (a). 


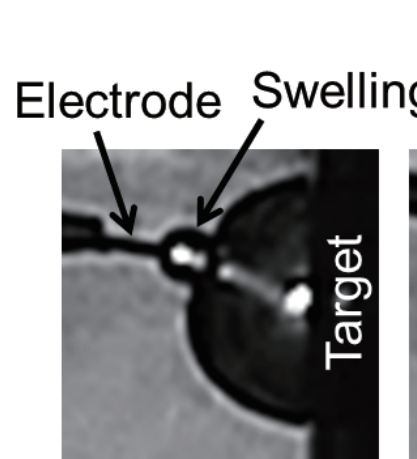

\section{Cavitation}
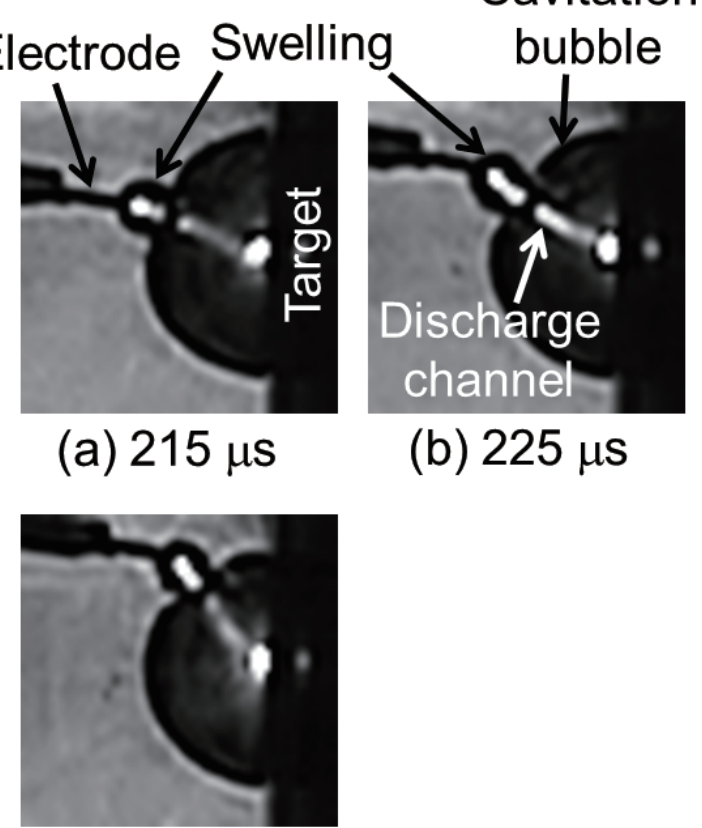

(b) $225 \mu \mathrm{s}$

(c) $200 \mu \mathrm{s}$

Figure 4. Discharge channels observed at three different positions of the electrode. 


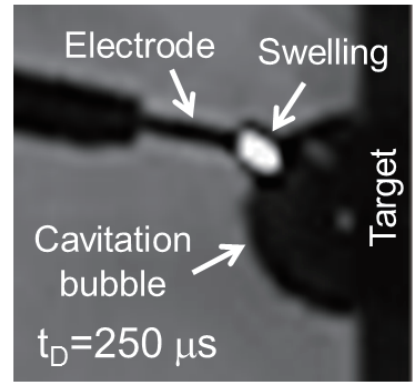

(a)

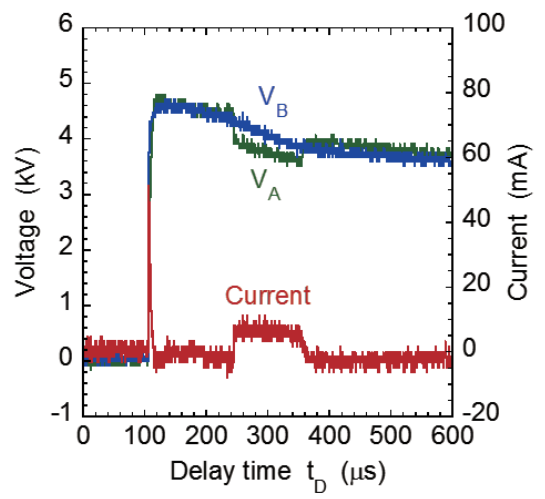

(b)

Figure 5. (a) Discharge channel and (b) voltage and current waveforms when an alumina target was employed. 


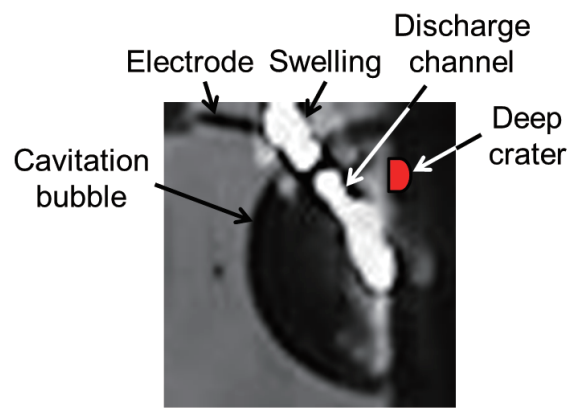

Figure 6. Discharge channel when an ablation crater was located at the red spot in the figure. 

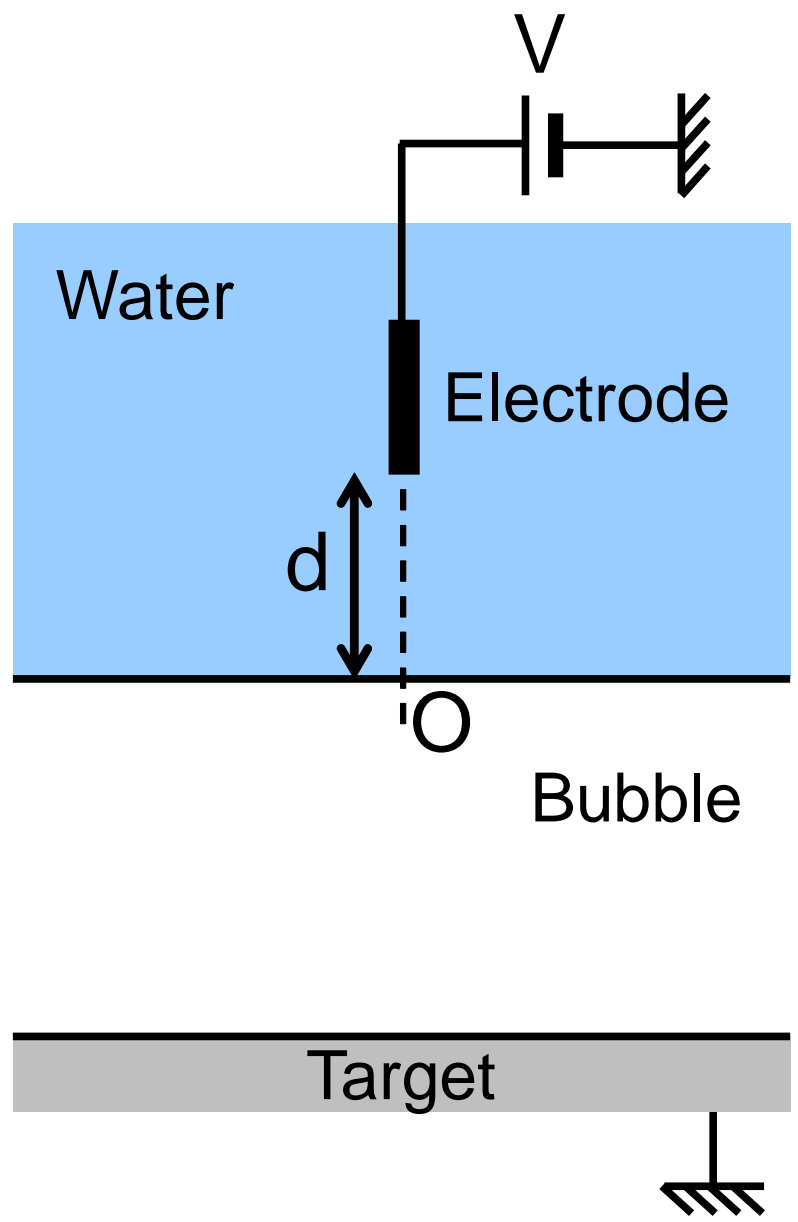

Figure 7. Simplified discharge geometry which was assumed in the theoretical calculation of the electrostatic pressure. 


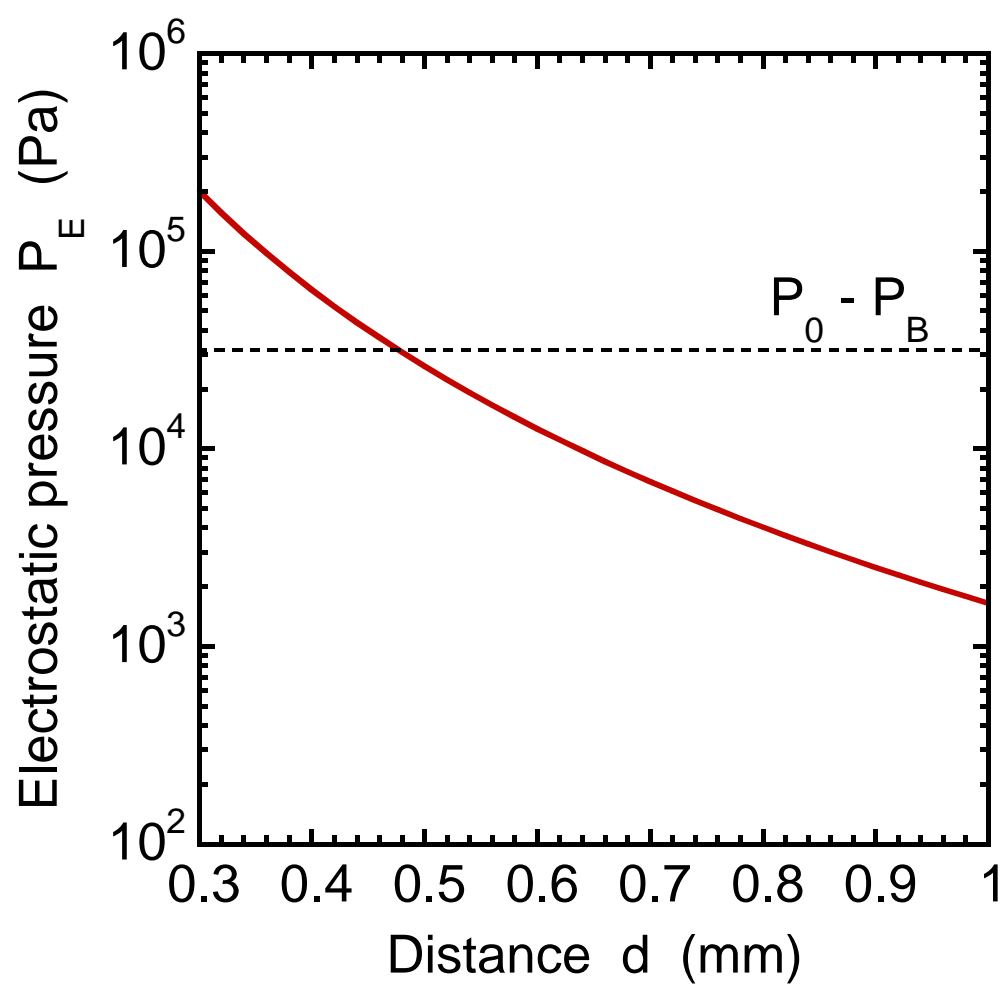

Figure 8. Electrostatic pressure as a function of the distance between the tip of electrode and the gas-liquid boundary of the cavitation bubble. The horizontal dotted line shows the pressure difference between the cavitation bubble and water. 